\title{
Interference Reduction Technique in Mobile Adhoc Networks using Mathematical Prediction Filters
}

\author{
G.Suseendran \\ Ph.D. Research Scholar \\ Department of Mathematics \\ Presidency College (Autonomous), Chennai-5
}

\author{
E.Chandrasekaran \\ Associate Professor \\ Department of Mathematics \\ Presidency College (Autonomous), Chennai-5
}

\begin{abstract}
Interference is one of the major degrading factors of mobile ad hoc networks (MANET). Since, MANET transmits over the single wireless channel; packets of the same flow interfere with each other greatly. This mutual interference causes the bottleneck over the network. Further, predicting interference accurately around a transmitter or receiver is a complicated task. As a solution to these problems, in this paper, we propose an interference reduction technique for MANET using mathematical prediction filters. The technique used Hidden Markov Model (HMM) for predicting the interference of the nodes. Initial transmission power is set by comparing received signal power with minimum and maximum values. During data transmission, RTS and CTS messages are exchanged using initial transmission power level and they include interference value of the source and destination respectively. The source then uses the interference value of the destination to transmit data packet. On the other hand, the destination uses the interference value of the source for transmitting the acknowledgement (ACK) packet to the source. Thus, this interference calculation at both ends reduces the interference. By simulation, we show that our technique reduces the interference considerably.
\end{abstract}

\section{Keywords}

Mobile Ad Hoc Networks (MANET), Hidden Markov Model (HMM, Interference Reduction, Prediction Filters, Power control

\section{INTRODUCTION}

\subsection{Mobile Ad hoc Networks (MANET)}

An infrastructure less, temporary network with multiple mobile nodes is defined as a wireless adhoc network. [1] Since, MANET encompasses of self-configuring and self- organizing mobile hosts; transmission is performed only through multihop connectivity. Non-existence of centralized administration, inadequacy of power and bandwidth, dynamic network topology are the characteristics of MANET. [2] Mobile nodes are endowed with wireless radio, memory, processor and power source. [3] Wireless adhoc networks have more characteristics that are special and some shortcoming when compared with wired networks. [4]

Wireless ad hoc networks make available multitude of nodes to inaugurate and maintain a network. In short, it supports selfhealing architecture without any base station or coordinator and this is the main trait of MANET. Due to meritorious attributes of wireless ad hoc networks such as processing capability and small transceivers, it is useful in many fields namely, disaster management, military applications, crisis response and so on. [5]

\subsection{Interference in MANET}

In MANET, transmission ranges are different from a device to device. This variation in transmission range commences the problem of interference. More interference is rooted by a device that has larger transmission range. Consequently, a device with smaller range may not be able to communicate with other device. [6]

Mutual interference reasoned by concurrent transmissions and intensity of the received signals confines the wireless networks. The primary performance limits of wireless ad hoc networks are determined by the interference. However, predicting the interference together with fading effects and node distributions is a puzzling problem. [7]

The throughput performance of MANET is drastically degraded by a bottleneck of mutual interference. To increase the network throughput, multiple antennas can be implemented at the radio nodes. [8]

\subsection{Interference Reduction}

Interference is one of the major degrading factors in MANET. Hence, reducing the level of interference to improve the network performance becomes an important task. Selecting appropriate transmission power is considered as an effective mechanism for reducing the interference level [10]. Employing multiple channels reduce the level of interference, as a result multi-channel communications increase the throughput of MANET when compare with single- channel communications [9]. Interference can also be reduced by the use of power control schemes. Even though power control schemes increase the system capacity, it revenues low signal to interference plus noise ratio (SINR) that is weak received signal. [10] To guarantee network-wide connectivity, power controlled topology management schemes try to find lowest transmission power level for each link [17].

The well-organized and flexible approach to select transmission rate is link adaptation, it chooses the transmission rate based on channel conditions. Network interference must be lessened to achieve distributed optimal local and global utility and to allow the users to transmit data at minimum transmission power, while still maintaining connectivity. [12]

The process of minimizing interference between network nodes can attain extensive energy conservation. Reducing the interference alleviates number of collisions and retransmissions on the MAC and consequently lowers energy consumption. Topology control scheme selects appropriate transmission power for each node and eliminates long-range connections thereby it reduces the interference. [3] 


\subsection{Problem Identification}

In [18], Bassel Alawieh et al. have presented a distributed correlative power control scheme for MANET. Their scheme has measured interference value for transmitting both control and data packets. This sequential interference prediction leads to overhead problems.

Further, initial transmission is sent at maximum transmission value, which shows demerit of their power control scheme. They have used kalman filter for predicting interference. Kalman filter is less accurate when compare with hidden markov model (HMM).

In this paper, we propose an interference reduction technique for MANET using mathematical prediction filters. The Hidden Markov Model (HMM) technique is used for predicting the interference of the nodes.

\section{RELATED WORKS}

Salam Akoum et al. [8] have proposed spatial interference mitigation at the transmitter for multiple input single output ad hoc networks. They have applied zero forcing beam forming at the transmitter, and have analyzed the corresponding network throughput and transmission capacity. By assuming a network with Poisson distributed transmitting nodes and spatially independent Rayleigh fading channels, they have applied mathematical tools from stochastic geometry to derive a lower bound on the probability of outage. Their approach has increased residual interference at the receiver.

V. Haghighatdoost et al. [11] have proposed a general algorithm called $A_{p l n}$ for finding the spanning tree of separate nodes in the plane. Their $A_{\text {pln }}$ algorithm presents an iterative routine for minimizing the maximum interference of the resulting spanning tree. At the beginning the resulting tree has only one edge, which is the smallest edge in the input graph, until all input nodes are not connected together, their algorithm adds a new edge to the resulting tree. For adding a new edge to sub graph the best edge, which imposes minimum increase on the interference of all nodes from all available edges is selected. Their $A_{\text {pln }}$ is a general algorithm for any two dimensional distribution and it has no limit or special conditions for the input distribution.

Fredrick Awuor et al. [12] have proposed coupled interference network utility maximization (NUM) strategy (i.e. CIN) for rate adaptation in WLANs that is solved using "reverseengineering" based on Karush-Kuhn-Tucker (KKT) conditions. According to their approach, the users determine data rates based on their local observations (i.e. coupled interference). Both pricing and limited message passing mechanisms are employed in the NUM wherein pricing restrict users from selfinterest behaviors while limited message passing assist users to announce their prices and transmit powers.

Nouha Jaoua et al. [13] have proposed an approach based on Bayesian estimation using particle filtering. They have proposed their approach to estimate jointly the multicarrier signal and the noise parameters. Based on sequential Monte Carlo (SMC) methods, their proposed scheme allows the online estimation using a Raoblackwellized particle filter.

Guinian Feng et al [14] have proposed a topology control algorithm called minimum interference algorithm (MIA), to minimize the overall network interference. They have formulated the pair wise interference condition between two links, and showed that the interference conditions for the minimum-transmit-power strategy and the equal-transmitpower strategy are equivalent. Based on the pair wise definition, they have further investigated the "typical" interference relationship between a link and all other links in its surrounding. They have defined a new metric called the interference coefficient to characterize the extent of the interference between a link and its surrounding link.

\section{INTERFERENCE REDUCTION IN MANET USING MATHEMATICAL PREDICTION FILTERS}

\subsection{Overview}

In this paper, we propose interference reduction technique in MANET using hidden markov model (HMM). Initially, node that receives the RREQ packet calculates its received signal power and compares with predefined threshold values namely, $\mathrm{P}_{\min }$ and $\mathrm{P}_{\max }$. Based on these comparison initial transmission values are set. During transmission, RTS message is sent using initial transmission power. RTS includes interference value predicted by the source. Nodes use hidden markov model (HMM) to predict their interference value. On receiving RTS, the destination calculates its interference and sends it along with CTS to the source with the power level $\mathrm{P}_{\text {ini. }}$. While receiving the CTS, the source calculates the minimum power required for transmitting data using the interference value of the destination and transmits the data with that power value. Finally, the destination uses the interference value of the source for transmitting the ACK message.

\subsection{Estimation of Received Signal Power}

Received signal power of a node can be calculated as, [18]

$$
r_{p}=t_{p} \times d^{-4} \times G^{2} \times h^{2} \times 10^{\tau / 10}
$$

Whereas, $r_{p}$ is the received power,

$$
t_{p} \text { is the transmitted power, }
$$

$d$ represents distance between any two nodes,

$G$ denotes node's omnidirectional antenna gain,

$h$ is the height of the antenna,

$\tau$ indicates shadowing component of Gaussian random variable with zero mean and standard deviation of $\sigma \mathrm{db}$.

\subsection{Evaluation of Signal to Interference plus Noise Ratio (SINR)}

During the transmission of data from a node $i$ to its destination, its signal to interference plus noise ratio (SINR) can be evaluated as follows. Assume, the data is transmitted with a constant power $\chi$ to its intended destination and both are positioned with a fixed D distance away, then its SINR is symbolized as below, [22]

$$
\operatorname{SINR}=\frac{\chi D^{-\beta}}{\sigma+\sum_{i} \chi d_{i}^{-\beta}}
$$

Where, $\mathrm{d}$ is the distance between the nodes, $\sigma$ is the channel noise and $\beta$ is the path loss attenuation factor, $\beta>2$.

\subsection{Estimation of Channel Loss Gain}

Channel loss gain between any pair of nodes can be calculated as follows, [18]

$$
G=r_{p} \mid t_{p}
$$

$r_{p}, t_{p}$ are the received and transmitted power respectively. 


\subsection{Hidden Markov Model (HMM)}

Hidden markov model (HMM) is a stochastic signal model that provides theoretical description of a signal processing system. An HMM encompass of recurrent finite-state Markov Chain, output symbols and a distribution over that alphabet for each transition in the Markov Chain. In HMM, the states and transitions are hidden and output can only be seen through symbols. [20] The set $\mathrm{S}=\left(\mathrm{S}_{1}, \mathrm{~S}_{2}, \cdots, \mathrm{S}_{\mathrm{N}-1}, \mathrm{~S}_{\mathrm{N}}\right)$ are hidden states, they are not visible but arbitrarily generates the mixture of observations. The probability of the succeeding state depends on the previous state. [21]

Since, HMM has strong mathematical structure and it works accurately; it became a powerful modeling tool and used in wide variety of applications. [19] With the framework of natural language processing (NLP), HMM is the solution for many problems such as part-of-speech tagging and noun-phrase chunking [15]. Apart form these applications, HMM is well versed in temporal pattern recognition such as speech, handwriting, gesture recognition, part-of-speech tagging, musical score following, partial discharges and bioinformatics [16].

\subsection{HMM based Interference Prediction Model}

Our mechanism use Hidden Markov Model to measure interference of the nodes. Generally, in HMM $S$ is the set of hidden states and $\mathrm{V}$ is the set of observation states. Hidden state $\mathrm{S}$ and observation set $\mathrm{V}$ are depicted below in Equation (4) and (5) respectively. [15]

$$
\begin{gathered}
S=\left(s_{1}, s_{2}, s_{3}, \ldots, s_{n}\right) \\
V=\left(v_{1}, v_{2}, v_{3}, \ldots, v_{m}\right)
\end{gathered}
$$

In our HMM based prediction model, hidden state $\mathrm{S}$ represents interference value of nodes and observation state $\mathrm{O}$ denotes signal to interference plus noise ratio (SINR). In figure-1 we have shown basic hidden markov model (HMM).

Let $\mathrm{S}$ be the set of hidden states (interference)

$$
S=I_{1}, I_{2}, . . I_{n}
$$

Let $\mathrm{N}$ be a fixed state sequence of length $\mathrm{T}$ given by,

$$
N=n_{1}, n_{2}, \ldots, n_{T}
$$

Let $\mathrm{O}$ be the corresponding observations given by,

$$
O=s n_{1}, s n_{2}, \ldots, s n_{T}
$$

HMM is generally formulated as, [15]

$$
\lambda=(A, B, \pi)
$$

Where, $A$ is a transition array and it is independent of time $t$. This array stores the probability of interference state $j$ following interference state $i$ and specified as below,

$$
A=\left\lfloor a_{i j}\right\rfloor a_{i j}=P\left(n_{t}=I_{j} \mid n_{t-1}=I_{i}\right)
$$

$\mathrm{B}$ is an array of observation, stores the probability of observation $k$, which is produced from the state $j$. Observation array is also independent of time $t$ and shown below,

$$
B=\left[b_{i}(k)\right], b_{i}(k)=P\left(x_{t}=s n_{k} \mid n_{t}=I_{i}\right)
$$

$\pi$ is the initial state probability,

$$
\pi=\left[\pi_{i}\right], \pi_{i}=P\left(n_{1}=I_{i}\right)
$$

Each node in the network measures its SINR value and passes it through the HMM model. Where, SINR is taken as observation state $\mathrm{O}$ and it is observed by the nodes with observing sequence $\mathrm{N}$.

Observation $(\mathrm{O})$ probability for a given sequence $\mathrm{N}$ is,

$$
P(O \mid N, \lambda)=\prod_{t=1}^{T} P\left(s n_{t} \mid n_{t}, \lambda\right)=b_{n 1}\left(s n_{1}\right) \times b_{n 2}\left(s n_{2}\right) . . b_{n T}\left(s n_{T}\right)
$$

The probability of the state sequence is given by,

$$
P(N \mid \lambda)=\pi_{n 1} a_{n 1 n 2} a_{n 2 n 3} \ldots a_{n T-1 n T}
$$

By using equation (12) and (13) we can easily estimate probability of the observations,

$$
\begin{aligned}
& P(O \mid \lambda)=\sum_{N} P\left(O \mid N_{1} \lambda\right) P(N \mid \lambda)=\sum_{n 1 \cdots n T} \pi_{n 1} b_{n 1}\left(s n_{1}\right) \\
& a_{n 1 n 2} b_{n 2}\left(S n_{2}\right) \cdots a_{n} T-1 n T^{b} n T(s n T)
\end{aligned}
$$

To discover the single best state sequence for an observation sequence $\mathrm{n}_{1}$, we use Viterbi algorithm. For measuring maximum likelihood state, we define the probability of the most probable path as,

$$
\vartheta(i)=\max _{n 1, n 2, n 3, \ldots . n T-1} P\left(\begin{array}{l}
n_{1}, n_{2} . . n_{T}=I_{i}, s n_{1}, \\
s n_{2} . . s n_{t} \mid \lambda
\end{array}\right)
$$

From the above equation (15), we can measure the highly likelihood state as,

$$
\stackrel{*}{n}=\arg \max _{1 \leq i \leq N}\left[\vartheta_{T}(i)\right]
$$

The sequence of states can be backtracked as the pointer in each state represents. State sequence backtracking is shown below,

$$
{ }_{t}^{*}=\Phi_{t+1}\left(\begin{array}{c}
* \\
n_{t+1}
\end{array}\right), t=T-1, T-2, \ldots 1 .
$$

Whereas, $\Phi$ is an additional matrix of size $\mathrm{N}^{*} \mathrm{~T}$, needs to be introduced in Viterbi algorithm to find the best path. T represents sequence length time. This backtracking gives the required set of states.

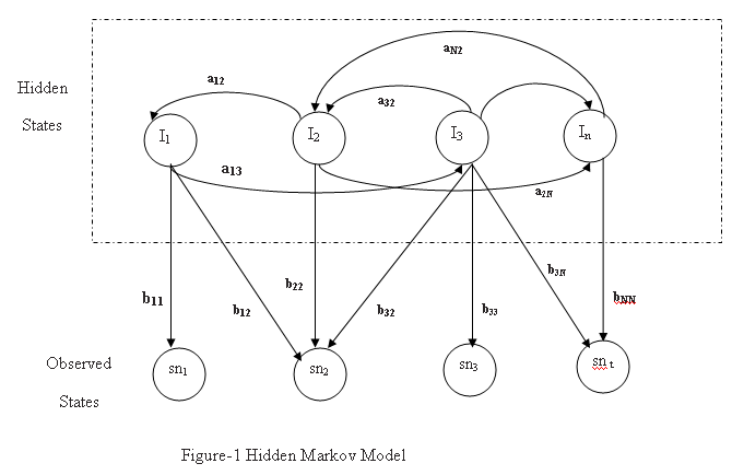

\subsection{Transmission Power Assignment Strategy}

\subsubsection{Initial Transmission Power Assignment}

When a node receives the route request (REQ) packet from the source, it measures its average received signal power (RSP) (using (1)). Then it compares its RSP with two predefined threshold values minimum received signal power $\left(\mathrm{RS}_{\min }\right)$ and maximum received signal power $\left(\mathrm{RS}_{\max }\right)$ and assigns its initial transmission power $\left(\mathrm{P}_{\mathrm{ini}}\right)$.

If $\left(\mathrm{RSP}>\mathrm{RS}_{\max }\right)$

Then 
Set $\mathrm{P}_{\text {ini }}=\mathrm{P}_{\text {min }}$

Else if $\left(\mathrm{RSP}<\mathrm{RS}_{\min }\right)$

Then

$$
\text { Set } \mathrm{P}_{\text {ini }}=\mathrm{P}_{\max }
$$

End if

End if

If the measured RSP is greater than $\mathrm{RS}_{\max }$, it shows that transmitting and receiving nodes are very close to each other. Thus, our algorithm sets $\mathrm{P}_{\text {ini }}$ to $\mathrm{P}_{\min }$. On the other hand, if RSP is lesser than $\mathrm{RS}_{\text {min }}$, it reveals either long distance between two nodes or more interference on that link. Therefore, its $\mathrm{P}_{\text {ini }}$ is set to $\mathrm{P}_{\max }$.

\subsubsection{Interference Aware Data Transmission}

If a node wants to transmit data to the destination, it first measures SINR (using (2) as per section 3.3) and passes into HMM prediction filter. As a result, the node receives predicted interference $\left(\mathrm{PI}_{1}\right)$ around its transmission zone. The source then encloses $\mathrm{PI}_{1}$ value in RTS message and sends at $\mathrm{P}_{\text {ini }}$ to the destination.

$$
\text { Node } \mathrm{i} \stackrel{R T S}{\longrightarrow} \text { Node } \mathrm{j}
$$

Upon receiving the RTS message, the destination node estimates the predicted interference $\left(\mathrm{PI}_{2}\right)$ around its transmission zone using $\mathrm{HMM}$ prediction filter. Then, it includes $\mathrm{PI}_{2}$ value in CTS message and sends at $\mathrm{P}_{\text {ini }}$ to the source node.

$$
\text { Node } \mathrm{i} \stackrel{\text { CTS }}{\longleftarrow} \text { Node } \mathrm{j}
$$

When the source receives the CTS message, it extracts the interference value $\left(\mathrm{PI}_{2}\right)$ from the CTS message and measures the $\mathrm{P}_{\min }$ value for sending data packets. The formula used by the source node is depicted below, [18]

$$
P_{\text {min }}=\max \left(P_{\text {ini }}, \kappa \times \frac{P I_{2}}{G}\right)
$$

Where, $\boldsymbol{K}$ is the predefined SINR threshold value and $\mathrm{G}$ represent channel loss gain which is calculated as mentioned in section 3.4

After the successful reception of data packets, the destination uses the $\mathrm{PI}_{1}$ value for measuring $\mathrm{P}_{\min }$ for sending ACK message to the source node. The formula is shown below, [18]

$$
P_{\min }=\max \left(P_{\text {ini }}, \kappa \times \frac{P I_{1}}{G}\right)
$$

Thus, the interference at both the source and the receiver is minimized.

The overall process of our interference reduction scheme is described below,

\section{Algorithm}

1. Node that receives RREQ packet calculates RSP (as per section 3.2)

2. The node compares its RSP with predefined threshold values $\mathrm{P}_{\min }$ and $\mathrm{P}_{\max }$

3. If the measured RSP $>P_{\max }$ then

$$
3.1 \mathrm{P}_{\text {ini }}=\mathrm{P}_{\max }
$$

Else If the measured RSP $<\mathrm{P}_{\min }$ then

End if

$$
3.2 \mathrm{P}_{\text {ini }} \text { is set to } \mathrm{P}_{\min }
$$

4. During data transmission, the source measures SINR (as per section-3.3) and pass through HMM prediction filter.
5. The source estimates the predicted interference $\left(\mathrm{PI}_{1}\right)$ around its transmission zone using HMM prediction filter.

6. Source encloses $\mathrm{PI}_{1}$ in RTS and it at power level $\mathrm{P}_{\text {ini }}$ to the destination.

7. On receiving RTS, the destination predicts interference $\mathrm{PI}_{2}$ around its transmission zone using HMM filter

8. Destination encloses $\mathrm{PI}_{2}$ with $\mathrm{CTS}$ and sends back to the source at power level $\mathrm{P}_{\text {ini. }}$.

9. The source uses the $\mathrm{PI}_{2}$ for calculating $\mathrm{P}_{\min }$ required for transmitting data packets to the destination. (As per equation18)

10. Upon successful reception of data packets, the destination use $\mathrm{PI}_{1}$ to calculate $\mathrm{P}_{\min }$ for sending ACK packet to the source node (As per equation-19)

\section{SIMULATION RESULTS}

\subsection{Simulation Parameters}

We evaluate our Interference Reduction Technique through NS2 [23]. We use a bounded region of $1000 \times 1000 \mathrm{sqm}$, in which we place nodes using a uniform distribution. The number of nodes is varied as $25,50,75$ and 100 . We assign the power levels of the nodes such that the transmission range of the nodes varies from 250 meters to 400 meters. In our simulation, the channel capacity of mobile hosts is set to the same value: 2 Mbps. We use the distributed coordination function (DCF) of IEEE 802.11 for wireless LANs as the MAC layer protocol. The simulated traffic is Constant Bit Rate (CBR).

The following table summarizes the simulation parameters used

Table 1: Simulation Parameters

\begin{tabular}{|c|c|}
\hline No. of Nodes & $25,50,75$ and 100. \\
\hline Area Size & 1000 X 1000 \\
\hline Mac & 802.11 \\
\hline Simulation Time & $50 \mathrm{sec}$ \\
\hline Traffic Source & CBR \\
\hline Packet Size & 500 \\
\hline Transmit Power & $0.660 \mathrm{w}$ \\
\hline Receiving Power & $0.395 \mathrm{w}$ \\
\hline Idle Power & $0.035 \mathrm{w}$ \\
\hline Initial Energy & $10.3 \mathrm{~J}$ \\
\hline Transmission Range & $250,300,350$ and $400 \mathrm{~m}$ \\
\hline Routing Protocol & AODV \\
\hline Flows & $2,4,6$ and 8. \\
\hline
\end{tabular}

\subsection{Performance Metrics}

We compare the performance of our proposed HMM prediction filter with Kalman Filter based prediction technique [18]. We evaluate mainly the performance according to the following metrics:

Average Packet Delivery Ratio: It is the ratio of the number .of packets received successfully and the total number of packets transmitted.

Average Energy Consumption: The average energy consumed by the nodes in receiving and sending the packets.

Estimation Error: It is the estimation error, which indicates how close the estimated interference values are close to the actual values.

\section{A. Based on Flows}

In our experiment we vary the number of CBR traffic flows as 2, 4, 6 and 8 with 50 nodes. 


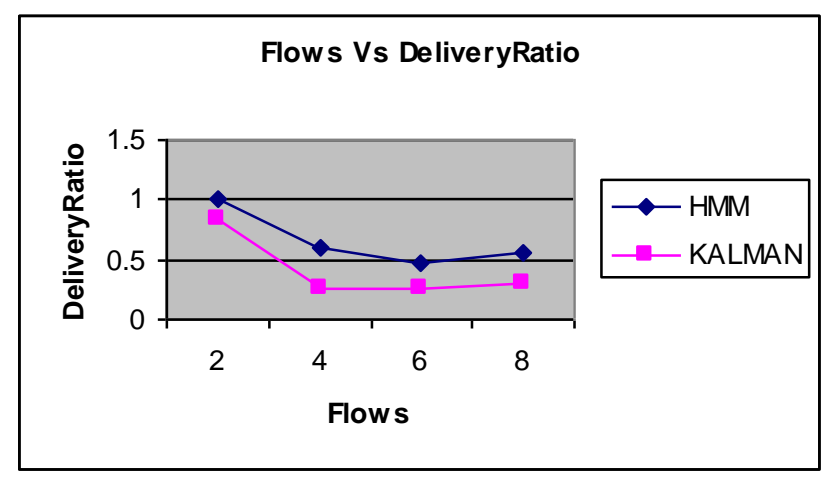

Figure 2: Flows Vs Delivery Ratio

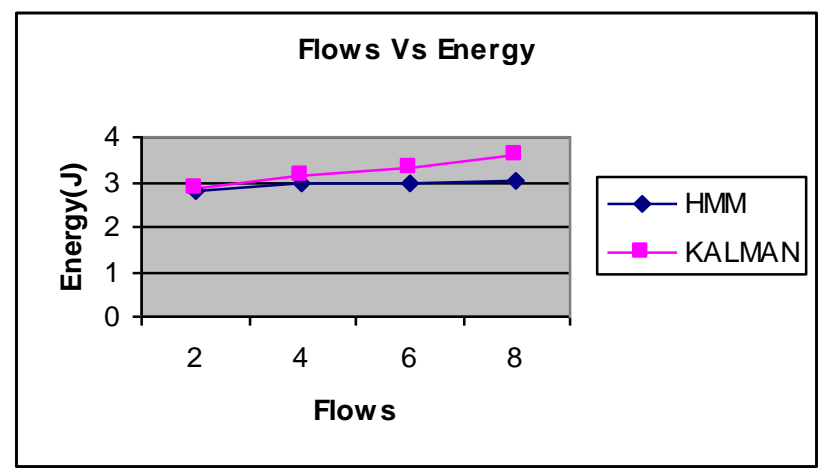

Figure 3: Flows Vs Energy

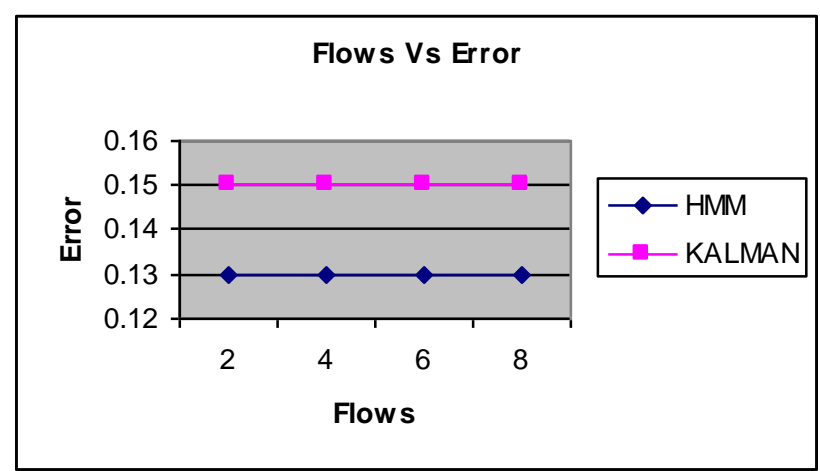

Figure 4: Flows Vs Error

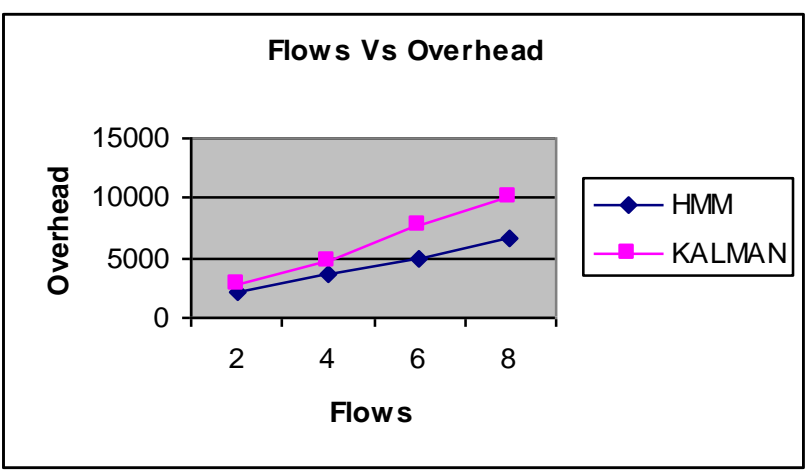

Figure 5: Flows Vs Overhead

When the number of traffic flows is increased, naturally the interference at the source and destination increases gradually, resulting in degradation of packet delivery ratio. Figure 2 gives the packet delivery ratio for various traffic flows. From the figure, it can be seen that HMM has slightly higher delivery ratio when compared to Kalman Filter scheme.

When number of traffic flows is increased, the energy consumption for transmitting and receiving is also increased. From figure 3, we can see that the energy consumption of our proposed HMM is less than the existing KALMAN technique, since the transmission power adjustment is accurately done with the help of predicted interference values.

From figure 4, we can see that the increase in traffic flows has no impact on the estimation error. But the estimated error of our proposed HMM filter technique is less than the KALMAN technique.

Figure 5 presents the overhead involved when the traffic flows are increased from 2 to 8 . Clearly the overhead increases since additional RTS and CTS messages have to be transmitted for each flow. We can see that the overhead of the proposed HMM technique is less than the existing KALMAN technique, since it eliminates the transmission power values for RTS and CTS packets.

\section{B. Based on Nodes}

In our second experiment we vary the number of nodes as 25 , 50,75 and 100 keeping the number of flows as 8 .

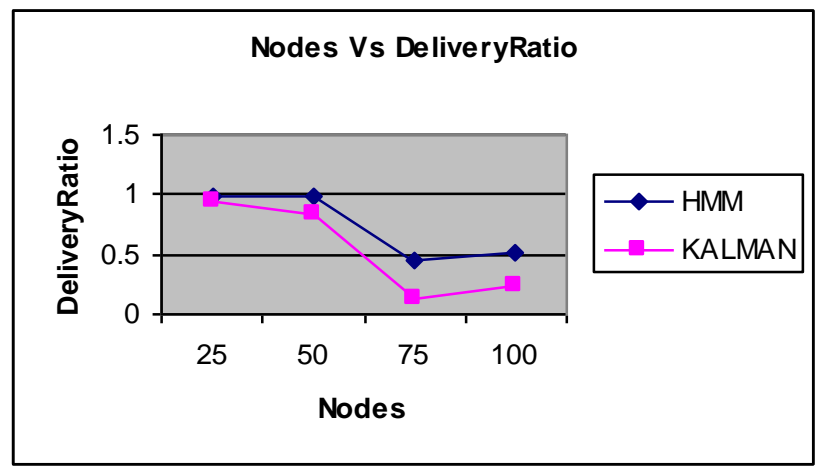

Figure 6: Nodes Vs Delivery Ratio

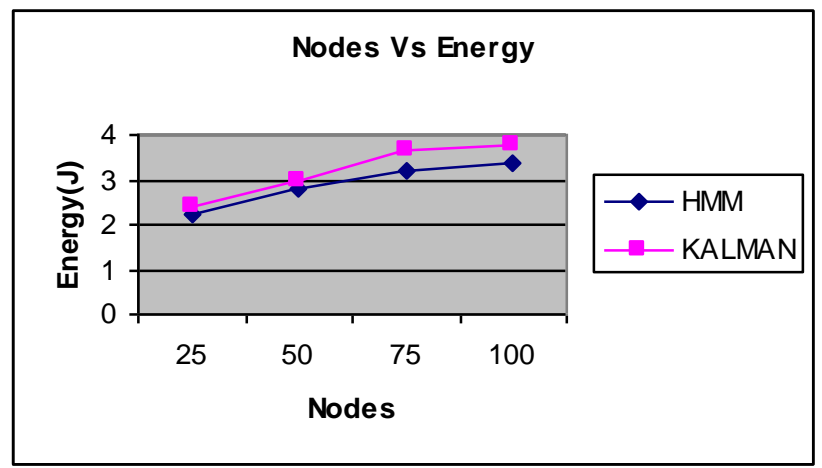

Figure 7: Nodes Vs Energy 


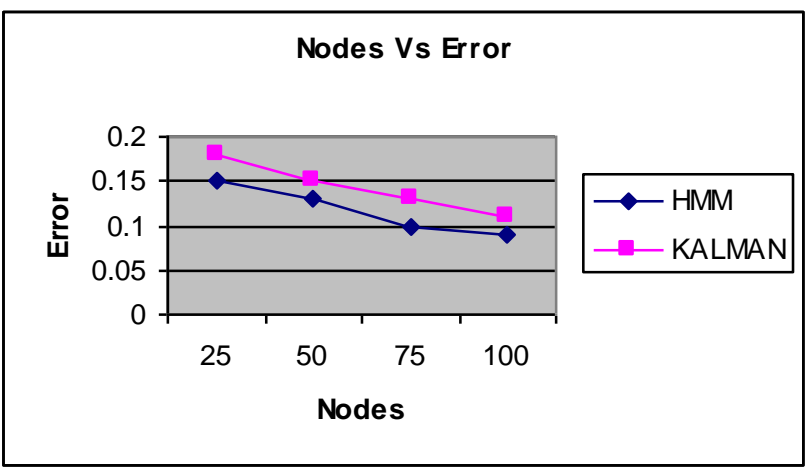

Figure 8: Nodes Vs Error

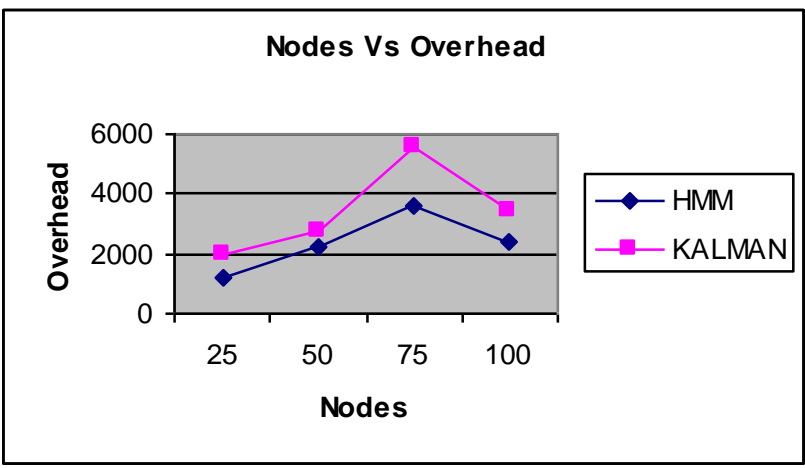

Figure 9: Nodes Vs Overhead

When the number of nodes is increased, naturally the interference around the source and destination increases gradually, resulting in degradation of packet delivery ratio. Figure 6 gives the packet delivery ratio for various traffic flows. From the figure, it can be seen that HMM has slightly higher delivery ratio when compared to Kalman Filter scheme.

When number of nodes is increased, the energy consumption for transmitting and receiving is also increased, since the path involves more number of hops. From figure 7, we can see that the energy consumption of our proposed HMM is less than the existing KALMAN technique, since the transmission power adjustment is accurately done with the help of predicted interference values.

Figure 9 presents the overhead involved when the number of nodes is increased from 2 to 8 . Clearly the overhead increases since more routing messages have to be transmitted. We can see that the overhead of the proposed HMM technique is less than the existing KALMAN technique, since it eliminates the transmission power values for RTS and CTS packets.

From figure 8 , we can see that the estimated error of our proposed HMM technique is less than the existing KALMAN technique.

\section{Based on Range}

In our third experiment we vary the transmission range as $250,300,350,400$ and 450 with 50 nodes and 8 flows.

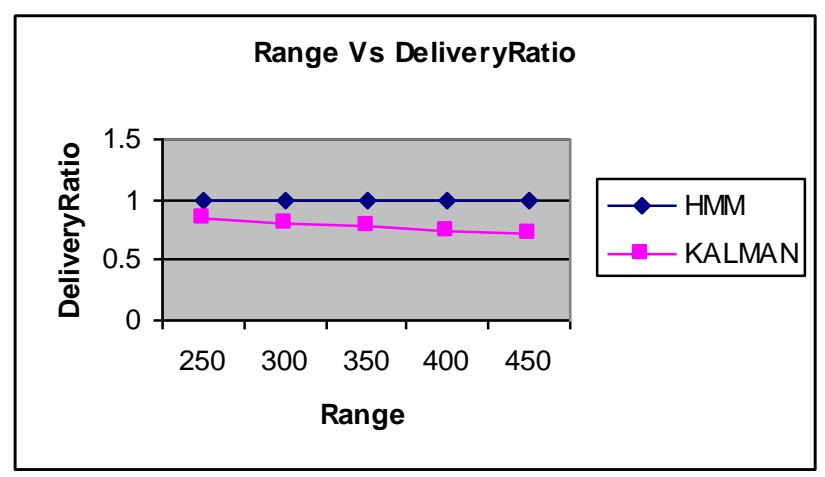

Figure 10: Range Vs Delivery Ratio

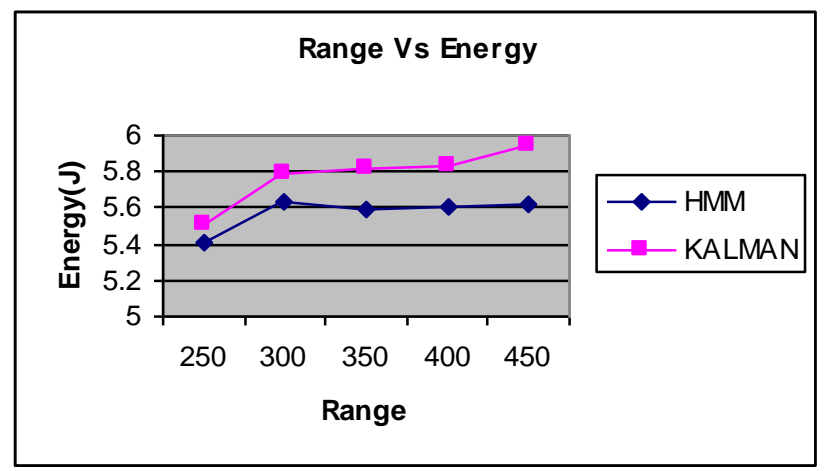

Figure 11: Range Vs Energy

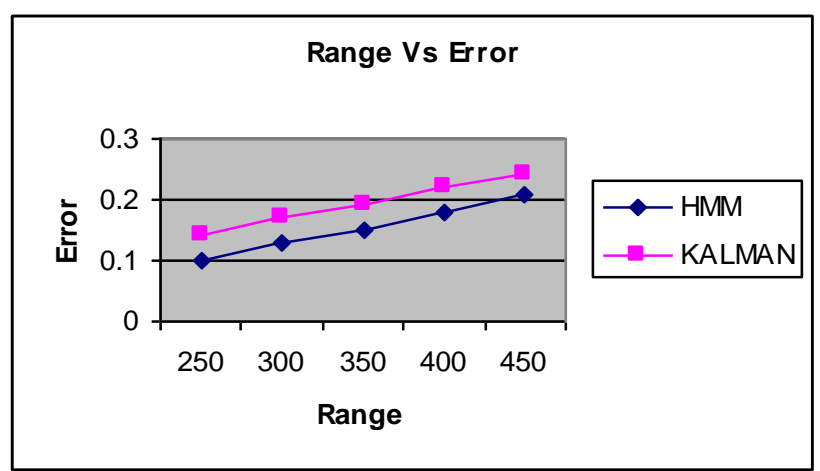

Figure 12: Range Vs Error

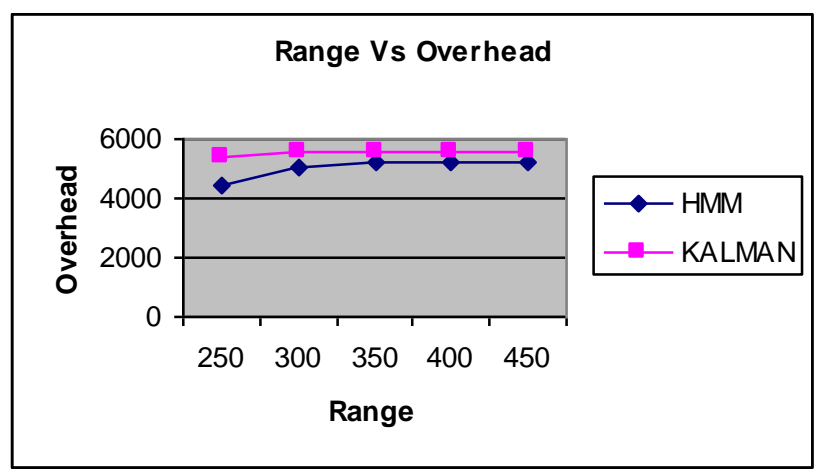

Figure 13: Range Vs Overhead

From figure 10, we can see that varying the range has less impact on packet delivery ratio for both the techniques. We can 
see that our proposed HMM technique has higher packet delivery ratio than the existing KALMAN technique.

When the transmission range is increased, the energy consumption for transmitting and receiving is also increased, since it involves more transmitting and receiving power. From figure 11 , we can see that the energy consumption of our proposed HMM is less than the existing KALMAN technique, since the transmission power adjustment is accurately done with the help of predicted interference values.

From figure 12, we can see that the estimated error of our proposed HMM is less than the existing KALMAN technique.

From figure 13, we can see that the overhead of our proposed HMM is less than the existing KALMAN technique.

\section{CONCLUSION}

In this paper, we have proposed an interference reduction technique for mobile adhoc network (MANET) using mathematical prediction filters. Our technique uses Hidden Markov Model (HMM) for predicting the interference of nodes. Initial transmission power is set by comparing the received signal power with minimum and maximum values. During data transmission, RTS and CTS messages are exchanged at initial transmission power values and they include the interference values of the source and destination, respectively. The source uses the interference value of the destination to transmit data packet. On the other hand, the destination uses the interference value of the source to transmit the ACK message. Thus, this interference calculation at both ends reduces the interference. We have shown the performance of our technique through simulations. Simulation results shows the proposed technique is better than the Kalman filter based prediction technique in terms of energy consumption, packet delivery ratio and prediction accuracy.

\section{REFERENCES}

[1] Wei Wei and Avideh Zakhor, "Interference Aware MultiPath Selection for Video Streaming in Wireless Ad Hoc Networks", IEEE Transactions on Circuits and Systems for Video Technology, Volume-19, Issue-2, pp-165 - 178, 2009

[2] H. X. Tan and W. K. G. Seah, "Dynamic Topology Control to Reduce Interference in MANETs", Proceedings of International Conference on Mobile Computing and Ubiquitous Networking (ICMU 2005), pp-13-15, 2005

[3] Pascal von Rickenbach, Stefan Schmid, Roger Wattenhofer, Aaron Zollinger, "A Robust Interference Model for Wireless Ad-Hoc Networks" Proceedings of IEEE International Parallel and Distributed Processing Symposium, (IPDPS'05), 2005.

[4] Kousha Moaveni-Nejad, Xiang-Yang Li, "LowInterference Topology Control for Wireless Ad Hoc Networks" Ad Hoc \& Sensor Wireless Networks, ACM Wireless Networks, 2005

[5] R. P. Ramos, M. Geandre R.ego, Tarciana Lopes, R. Baldini Filho and C. de Almeida, "Interference Evaluation in CDMA Ad Hoc Networks", IEEE International Telecommunications Symposium, pp- 967 - 970 , 2006

[6] Deepesh Man Shrestha, Guen-Hee Cho, Young-Bae Ko, We Duke Cho, "An Interference-aware Algorithm for Topology Control in Ubiquitous Ad hoc Networks" Proceedings of the Workshops in the Int'l Conf. on Ubiquitous Computing (Ubicomp'05), 2005.
[7] Roland Tresch, Giusi Alfano , Maxime Guillaud, “ Interference Alignment in Clustered Ad hoc Networks: high Reliability Regime and per-Cluster Aloha" IEEE International Conference on Acoustics, Speech and Signal Processing (ICASSP), pp- 3348 - 3351, 2011

[8] Salam Akoum, Marios Kountouris, M'erouane Debbahz, and Robert W. Heath, "Spatial Interference Mitigation for Multiple Input Multiple Output Ad Hoc Networks: MISO Gains", in proceedings of 45th Annual Asilomar Conference on Signals, Systems, and Computers, Pacific Grove, CA, Nov. 2011.

[9] Jian Tang, Guoliang Xue and Weiyi Zhang, "InterferenceAware Topology Control and QoS Routing in MultiChannel Wireless Mesh Networks" Proceedings of the 6th ACM international symposium on Mobile ad hoc networking and computing, (MobiHoc '05), 2005

[10] Katsuhiro Naito, Kazuo Mori, and Hideo Kobayashi, "Proposal of interference reduction routing for ad-hoc networks", Journal of Systemics, Cybernetics and Informatics, Vol- 8, No-5, pp. 28-33, 2010

[11] V. Haghighatdoost, M. Espandar, "A General Approach for Minimizing the Maximum Interference of a Wireless Ad-Hoc Network in Plane" International Journal of Computers, Communications \& Control, Vol-7, No-2, pp. 231-243, 2012

[12] Fredrick Awuor, Karim Djouani, Guillaume Noelz and Thomas Olwal, "Coupled Interference Based Rate Adaptation in Ad Hoc Networks" IEEE Conference AFRICON, pp-1-6, 2011

[13] Nouha Jaoua, Emmanuel Duflos, Philippe Vanheeghe, Laurent Clavier, Franc, ois Septier, “ Impulsive Interference Mitigation In Ad hoc Networks Based on Alpha-Stable modeling and Particle filtering" IEEE International Conference on Acoustics, Speech and Signal Processing (ICASSP), pp-3548 - 3551, 2011

[14] Guinian Feng, Soung Chang Liew, Pingyi Fan, "Minimizing Interferences in Wireless Ad Hoc Networks through Topology Control" Proceedings of IEEE International Conference on Communication Society (ICC'08), 2008

[15] Phil Blunsom, "Hidden Markov Models" The University of Melbourne, Department of computer science, digital.cs.usu.edu, 2004

[16] http://en.wikipedia.org/wiki/Hidden_Markov_model

[17] Kichan BAE, Geunhwi LIM, Namgi KIM, and Hyunsoo YOON, "Power Control in Wireless Ad Hoc Networks for Energy Efficient Routing with Capacity Maximization" Journal of Systemics, cybernatics, informatics, 2007

[18] 18. Bassel Alawieh , Chadi Assi, Wessam Ajib, "A Distributed Correlative Power Control Scheme for Mobile Ad hoc Networks using Prediction Filters" IEEE Transactions on Vehicular Technology, Volume- 57, Issue- 3, pp-1733 - 1744, 2008

[19] Jun Liu, Ibrahim Matta and Mark Crovella, "End-to-End Inference of Loss Nature in a Hybrid Wired/Wireless Environment", in Modeling and Optimization in Mobile, Ad Hoc and Wireless Networks (WiOpt), France,. 2003.

[20] Vasiliki L. Kakali, Panagiotis G. Sarigiannidis, Georgios I. Papadimitriou, and Andreas S. Pomportsis, "A Novel 
HMM-Based Learning Framework for Improving Dynamic Wireless Push Systems Performance", In Proceedings of Computers \& Mathematics with Applications, pp- 474-485, 2011

[21] Rahul Khanna and Huaping Liu, "System Approach to Intrusion Detection Using Hidden Markov Model", Proceedings of the international conference on Wireless communications and mobile computing, (IWCMC '06), 2006
[22] Mariam Kaynia, Geir E. Øien, and Nihar Jindal, "Joint Transmitter and Receiver Carrier Sensing Capability of CSMA in MANETs", IEEE Wireless Communications \& Signal Processing, (WCSP 2009), pp-1-5, 2009

[23] Network Simulator: http:///www.isi.edu/nanam/ns 